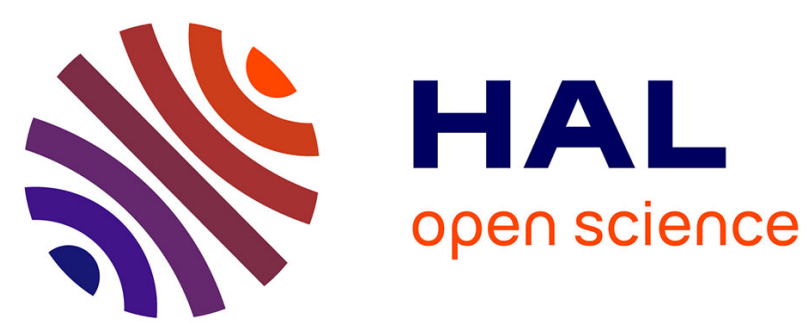

\title{
RECENT RESULTS ON THE STRUCTURE OF DISLOCATIONS IN TETRAHEDRALLY COORDINATED SEMICONDUCTORS
}

\author{
P. Hirsch
}

\section{- To cite this version:}

P. Hirsch. RECENT RESULTS ON THE STRUCTURE OF DISLOCATIONS IN TETRAHEDRALLY COORDINATED SEMICONDUCTORS. Journal de Physique Colloques, 1979, 40 (C6), pp.C6-27-C6-32. 10.1051/jphyscol:1979606 . jpa-00219022

\section{HAL Id: jpa-00219022 https://hal.science/jpa-00219022}

Submitted on 1 Jan 1979

HAL is a multi-disciplinary open access archive for the deposit and dissemination of scientific research documents, whether they are published or not. The documents may come from teaching and research institutions in France or abroad, or from public or private research centers.
L'archive ouverte pluridisciplinaire $\mathbf{H A L}$, est destinée au dépôt et à la diffusion de documents scientifiques de niveau recherche, publiés ou non, émanant des établissements d'enseignement et de recherche français ou étrangers, des laboratoires publics ou privés. 


\title{
RECENT RESULTS ON THE STRUCTURE OF DISLOCATIONS IN TETRAHEDRALLY COORDINATED SEMICONDUCTORS
}

\author{
P.B. Hirsch \\ Department of Metallurgy and Science of Materials, University of Oxford, Parks Road, Oxford, U.K.
}

Résumé.- Cet article décrit : a) des modēles structuraux de dislocations dissociées dans leur plan de glissement dans les semiconducteurs à coordination tétrahédrique, et corrèle ces structures avec les propriétés électriques - b) un mécanisme de montēe pour la transformation d'un défaut d'empilement intrinsèque dans une dislocation dissociée, en un défaut d'empilement extrinsẽque - c) les situations où les interactions coulombiennes entre partielles deviennent importantes - d) des résultats rêcents sur la dissociation des dislocations dans GaAs.

Abstract.- This paper describes : a) structural models of dislocations dissociated on glide planes in tetrahedrally coordinated semiconductors, and correlates these structures with electrical properties - b) a climb mechanism for the transformation of the intrinsic stacking fault bounded by partial dislocations in a dissociated dislocation, into an extrinsic stacking fault - c) the conditions under which coulomb forces between partials may be important - d) recent results on the dissociation of dislocations in GaAs.

1. Introduction.- Since the development of the weak beam technique of electron microscopy $/ 1 /$, it has been established without ambiguity that the dislocations in $\mathrm{Si}$ and $\mathrm{Ge}$, deformed under normal conditions, are dissociated into partials over most of their lengths $/ 2-5 /$, although some constricted segments have also been observed. Similarly, dislocations in III $V$ compounds have been shown to be dissociated /6-9/. Furthermore, "in situ" experiments in the electron microscope have shown glide of partial dislocations in Ge /10/, and of dissociated dislocations in Ge and $S i$ without prior constriction /11/.

It appears therefore that experimental data on physical properties of dislocated semiconductor crystals should be interpreted in terms of dissociated dislocations and that band structure calculations should be performed on models of such configurations.

In this paper we a) describe some structural models of dislocations dissociated on the glide planes (the simplest type of dissociated dislocations - the dissociated shuffle dislocations introduced by Hornstra /12/ and Haasen and Seeger /13/ wi1l not be discussed) - b) attempt to correlate structure with electrical properties - c) discuss the nature and origin of extrinsically dissociated and anomalously wide dislocations with screw components - d) examine the conditions under which coulomb forces between partials may become important e) report on recent results on the dissociation of dislocations in GaAs.
2. Structure of dislocations.- 2.1. 60 $0^{\circ}$ dis $\mathbf{l}$ oca tions.- Ball and spokes models have been constructed of dislocations of various orientations. Figure 1 shows the Thompson tetrahedron used for the dislocation $\mathrm{CB}$ on $\delta$ examined; $\mathrm{D}$ is below the plane of the paper.
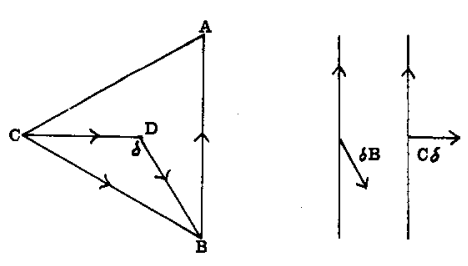

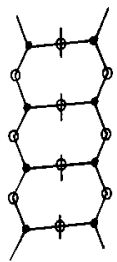

$\boldsymbol{b} \mathbf{B}$

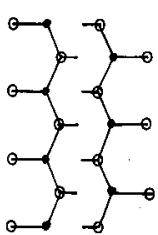

c)
Fig. 1 : Thompson tetrahedron and essential features of the core structures of the $90^{\circ}(C \delta)$ and $30^{\circ}(\delta B)$ partials of a dissociated $60^{\circ}$ dislocation (CB). Full and open circles refer to atoms on different, neighbouring (111) planes. D is below the plane of the paper.

The structures of the cores of the $30^{\circ}(\delta \mathrm{B})$ and $90^{\circ}$ (Co) are also shown. The dots and open circles represent atoms on the two neighbouring closely spaced (111) planes between which glide takes place and stacking faults are formed. The approximate directions of the dangling bonds, which for partials are approximately parallel to the glide planes (unlike the dangling bonds for undissociated dislocations 114/), are indicated by short lines. Figure 2 shows 
the same dislocations in the model, in which the top half of the crystal has been removed, and the bottom half retained but not shown below the planes containing the dislocation cores. There is an intrinsic fault between the two partials. Each of the partials in figure 2 is shown with a kink in it.

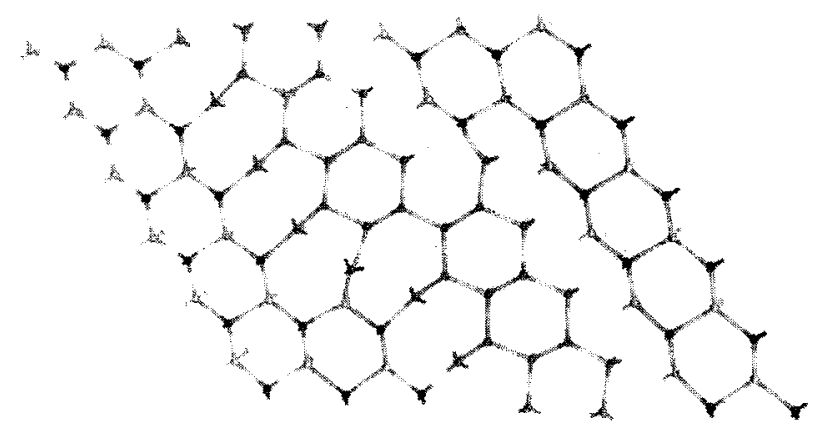

Fig. 2 : Dissociated $60^{\circ}$ dislocation. Core structure of $90^{\circ}$ partial on the right, and of the $30^{\circ}$ partial on the left, viewed normal to the slip plane. The top half of the crystal has been removed, and the bottom half retained, but only the planes containing the dislocation cores are shown. There is an intrinsic fault between the two partials. Kinks are shown on both partials.

The arrangement of the atoms along the $30^{\circ}$ partial in figure 2 (or the right hand one in figure 3) suggests $s p^{2} d$ rather than $s p^{3}$ hybridisation. The distance between atoms along the core is $b_{T}$, the second nearest neighbour distance, and equal to the magnitude of the total Burgers vector. An alternative structure is shown on the left hand side of figure 3 , in which neighbouring atoms along the core have been joined by single bonds; there are no dangTing bonds in this structure.

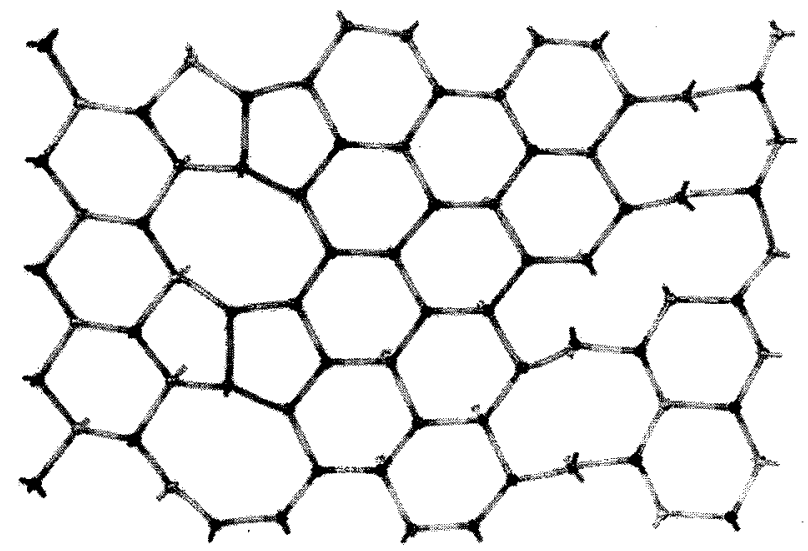

Fig. 3 : Dissociated screw dislocation, viewed normal to the slip plane. Only the planes containing the core structures of the two $30^{\circ}$ partials are shown. Two alternative core structures of the $30^{\circ}$ partial are shown. The right hand partial is shown with a kink in it.
The distance between atoms across the core of the $90^{\circ}$ partial is on $7 y 25 \%$ greater than the normal nearest neighbour distance. Bonding across the core is therefore likely. It is possible in principle to join the atoms across the core of the $90^{\circ}$ partial in pairs forming single bonds, but resulting in considerable distortion, and the symmetrical structure shown in figure 2 seems quite likely, with hybrid orbitäls being formed between both neighbours across the core.

2.2. Screw dislocation.- The screw consists of two $30^{\circ}$ partials, al ternative structures of which are shown in figure 3 , and which have already been discussed above. The $30^{\circ}$ partial on the right of figure 3. shows a kink.

2.3. Edge dislocation.- The edge dislocation consists of two $60^{\circ}$ partials, al ternative structures of which are shown in figure 4 for the two partials bounding the fault. The $60^{\circ}$ partial can be considered as made up of kinks in the $90^{\circ}$ partial (see Fig. 2), or in the $30^{\circ}$ partial (see Fig. 3 ). The position of one of the three atoms making up the repeat unit along the core of the $60^{\circ}$ partial is uncertain; alternative configurations are shown on the left and right hand partials. Similar alternative positions apply to the corresponding atoms in the kink of the $90^{\circ}$ partial (Fig. 2) and of the $30^{\circ}$ partial (Fig. 3); in both these cases the alternative shown for the $r . h .60^{\circ}$ partial in figure 4 has been shown.

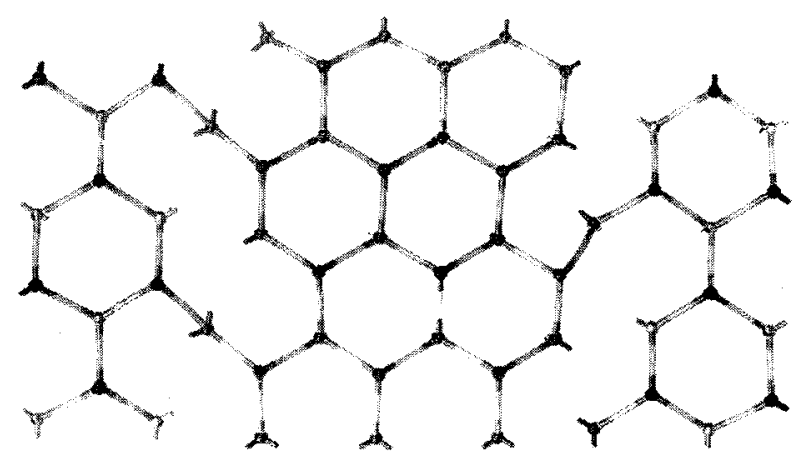

Fig. 4 : Dissociated edge dislocations consisting of two $60^{\circ}$ partials, viewed normal to the slip plane. Two alternative core structures are shown.

3. Correlation of structure with electronic properties.- Electrical measurements at low temperatures in $\mathrm{n}$ and $\mathrm{p}$ type $\mathrm{Ge}$, deformed in compression or torsion, have been interpreted successfully Labusch and Schettler $/ 15 /$, by Labusch and Schröter /16/ and Haasen and Wagner /17/ in terms of : 1) for screws a full donor band at $0.035 \mathrm{ev}$, and an empty acceptor band at $0.59 \mathrm{eV}$ above the valence band - 2) for edge 
dislocations a half full band in the neutral state at $0.09 \mathrm{eV}$ above the valence band. For $\mathrm{Si}$ the band for edge dislocations lies at $0.34 \mathrm{eV}$ above the valence band.

Since the screw consists of two $30^{\circ}$ partials we suggest that the $30^{\circ}$ partia) is associated (in the neutral state) with the full donor band close to the valence band and the empty acceptor band close to the conduction band. This behaviour would be consistent either with the structure shown on the 1.h.s. of figure 3 , in which there are no dangling bonds, the states near the conduction and valence bands being presumably associated with shear and dilational strainfields $/ 18 /$, or with the r.h.s. structure of figure 3 , and that of the 1.h. partial in figure 2, assuming there to be a large MottHubbard gap between the donor and acceptor states $/ 19,20 \%$.

The half full band at $0.09 \mathrm{eV}$ for Ge and 0.34 $\mathrm{eV}$ for $\mathrm{Si}$ is presumably associated with the $60^{\circ}$ partials constituting the edge dislocation, since edge dislocations are the major type of dislocations found in compressed specimens /21/. This is consistent with the structure for the $60^{\circ}$ partial shown in figure 4 ; there are three electrons per repeat unit consistent with a full and half full band.

This leaves open the problem of the electrical properties of the $90^{\circ}$ partial, one of the partials making up the $60^{\circ}$ dislocation. The periodicity of the structure in figure 2 is $b_{T}$, but there are two electrons per cell, suggesting a full band. But electrons with $k$ vectors along the dislocation Tine are not reflected by planes spaced at $b_{T}$ because of contributions from atoms at $\mathrm{b}_{\mathrm{T}} / 2$. This suggests that for the undistorted structure there is no energy gap at the first Brillouin zone boundary of the one dimensional band, and the band could appear effectively half fu11. However, the shear strain due to the $30^{\circ}$ partial destroys the additional symmetry, resulting in a gap. In any case, a Mott-Hubbard gap could occur, or the atoms may be joined in pairs by single bonds; in either case a gap would exist between any donor and acceptor states.

It should be noted that the atomic structure at a kink in a $90^{\circ}$ partial (see Fig. 2), and at one of the two types of kinks in the $30^{\circ}$ partial (see Fig. 3) is similar to that of a repeat period in an edge dislocation. This suggests that localised states at such kinks may have energy levels similar to those in the band associated with edge dislocations.
The structure of the second type of kink in a $30^{\circ}$ partial (see Fig. 2) seems however to be different. 4. Structure of extrinsically dissociated dislocations.- Gomez, Cockayne, Hirsch and Vitek /5/ have reported that in both Ge and Si, dislocations with screw character, generated by deformation at high temperature, are often dissociated into extrinsic faults, and that these dislocations have anomalously large widths. It has been assumed in the past that extrinsically dissociated dislocations are generated from intrinsically dissociated dislocations by a reaction of the type :

$$
\begin{aligned}
& \text { on a glide plane } \underline{C B}=\underline{C \delta}+\underline{\delta B} \\
& \text { on a neighbouring } 0=\underline{A \delta}+\underline{\delta A} \\
& \text { plane } \\
& \text { combined } \\
& \begin{aligned}
\underline{C B} & =(\underline{C \delta}+\underline{A \delta})+(\delta B+\delta A) \\
& =\underline{\delta}+\underline{C \delta}
\end{aligned}
\end{aligned}
$$

i.e. the effective position of the two partials $C \delta$, $\underline{\delta B}$ is reversed by nucleating $\underline{A \delta}+\underline{\delta A}$ on the neighbouring plane. An alternative configuration is formed as follows. Suppose vacancies (or interstitials) nucleate a dislocation loop, Burgers vector $B A$ on $\delta B$ of the screw dislocation $C B$ in figure $5 a, b$. On the p]ane of the intrinsic fault $\underline{B A}+\underline{B}=\underline{\delta A}$ and on $a$ parallel plane containing the top of the loop, $B A$ dissociates into $\underline{B \delta}$ plus $\delta A$ (Fig. $5 \mathrm{C}$ ); the latter sweeps across the plane to lie above the other partial $\mathrm{C} \delta$ (Fig. 5d). If this process occurs on adjacent glide planes an extrinsic fault will be formed.

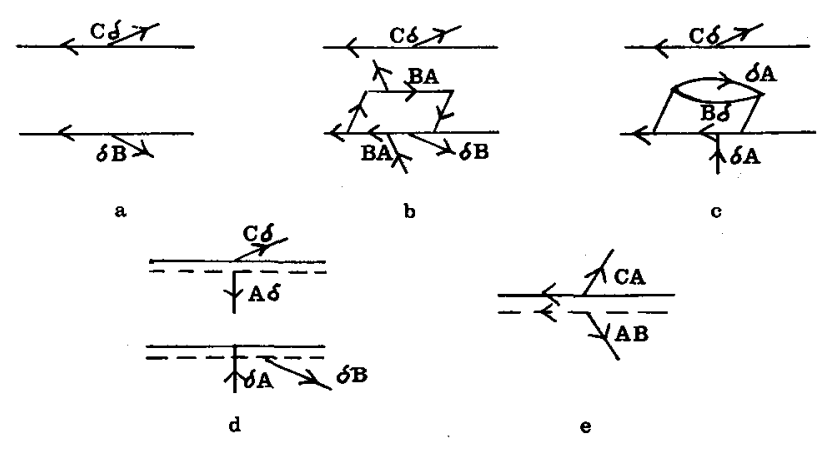

Fig. 5 : Mechanism of $c$ limb of dissociated screw $C B$, forming an extrinsically dissociated dislocation. See text for details.

The mechanism can still occur on a more distant parallel plane, but the total energy of the two faults will increase from that for the extrinsic fault $\left\langle\gamma_{E}\right\rangle$ to that of two overlapping intrinsic faults $\left(2 \gamma_{j}\right)$ with a consequent narrowing of the dislocation ribbon. The difference between this configuration of an extrinsically dissociated dislocation (produced by climb) and that produced by the glide mechanism 
is that two of the partials making up a pair bounding one side of the fault interchange planes (i.e. $\delta B$ and $\delta A$ in eq. 2, 3 and Fig. 5d). The dislocation can also be considered as consisting of two $60^{\circ}$ dislocations $C A$ and $A B$ overlapping on neighbouring planes. In practice the loop $B A$ is probably nucleated at a node where $\underline{\delta B}$ has pronounced edge character.

This mechanism of formation of extrinsic faults appears more plausible than that suggested tentatively in (5); the driving force for the climb mechanism of the intrinsic $\rightarrow$ extrinsic transformation is then a supersaturation of vacancies or interstitials on cooling from the high temperature of deformation. Recent experiments by Gomez have shown that when $\mathrm{Si}$ is cooled very slowly from about $820^{\circ} \mathrm{C}$ no extrinsic faults are formed, consistent with the cl imb mechanism.

5. Coulomb forces between partial dislocations.Using a mode 7 in which the charge on the partial dislocation is distributed uniformly over a cylinder, of radius $R$, and is screened by free carriers (electrons and holes), and assuming that the potential energy eV at the dislocations < kT, it can be shown that the electrostatic energy per unit length of the dissociated distocation is

$$
\begin{aligned}
E_{e} & =\frac{Q_{1}{ }^{2}}{\varepsilon}\left(\log _{R} \frac{\lambda}{R}-\frac{1}{2}\right)+\frac{Q_{2}{ }^{2}}{\varepsilon}\left(\log _{\frac{\lambda}{R}}-\frac{1}{2}\right)+ \\
& +\frac{2 Q_{1} Q_{2}}{\varepsilon}\left(\log _{D} \frac{\lambda}{D}-\frac{1}{2}\right)
\end{aligned}
$$

where $Q_{1} Q_{2}$ are the charges per unit length along the dislocation, $\varepsilon$ the dielectric constant and $\lambda$ (assumed $>$ R) the Debye screening length, i.e. $\lambda^{2}=k T \varepsilon / 4 \pi e^{2}(n+p)$

where $e$ is the electronic charge, $n, p$ the densities of electrons and holes away from the charged dislocation, $D$ is the separation of the two partials. The term in $E_{e}$ involving $Q_{1} Q_{2}$ occurs only if $\lambda>D$, i.e. below critical temperature $T_{C} \sim 500^{\circ} \mathrm{C}, 800^{\circ} \mathrm{C}$ for $\mathrm{Ge}$, Si respectively, and leads to a Coulomb force (per unit length) between partials

$F_{C}=\frac{2 Q_{1} Q_{2}}{\varepsilon D}$

The separation of the partials in then given by

$D=D_{0}+\frac{2 Q_{1} O_{2}}{\varepsilon Y}$

where $D_{0}$ is the equilibrium separation in the absence of a Coulomb force. Extending the treatment of Read /22/ and Labusch and Schröter /16/ for noninteracting electrons to dissociated dislocations, $Q_{1}, Q_{2}$ are controlled by the position of the Fermi Energy $E_{F}$ relative to the energy levels $E_{1}, E_{2}$ for the donor/acceptor bands for the two partials, i.e.

$$
\begin{aligned}
E_{F}= & E_{1}+\frac{2 e Q_{1}}{\varepsilon}\left(\log \frac{\lambda}{R}-\frac{1}{2}\right)+\frac{2 e Q_{2}}{\varepsilon}\left(\log \frac{\lambda}{D}-\frac{1}{2}\right)+ \\
& k T \log \frac{f_{1}{ }^{1}}{1-f_{1}{ }^{1}}
\end{aligned}
$$

and a similar expression for $E_{2}$, with $Q_{1}$ and $Q_{2}$ interchanged, and $f_{1}{ }^{1}$ replaced by $f_{2}{ }^{1} . f_{1}{ }^{1}=f_{1}$, $\frac{1}{2}+\frac{1}{2} f_{1}, 1+f_{1}$ for empty, half full and full bands respectively, where $f_{1}$ is the fraction of the states in the band occupied by electrons; $f_{1}$ is negative for holes in a full band. Expressions similar to (9) apply to all bands associated with the dislocations; the parameters $f_{j}$, and corresponding $E_{j}$, apply to the occupation of the particular band considered; however $Q_{1}, Q_{2}$ are the net line charges summing over all the possible states (electrons and holes) associated with the dislocation.

$Q_{1}, Q_{2}$ increase with increasing temperature, as $\lambda$ decreases; the maximum Coulomb force is expected to occur for temperatures just below $T_{c}$; for intrinsically dissociated dislocations, and for intrinsic materials. We expect the coulomb force to be a inaximum for edge dislocations. If $R=b_{T}$, we find for Ge at $700 \mathrm{~K}, f_{1}=f_{2} \approx 0.12$, and with $Q_{1}=Q_{2}=$ $\frac{2 e f_{1}}{\sqrt{3 b}}$, the coulomb force is found to be negligible. However, if the dislocations are surrounded by clouds of charged point defects or impurities, possibly bound also to the fault, the total charge on the dislocation and its associated cloud can be considerably greater, and the coulomb force can become important. Thus, if the point defects have donor levels very close to the valence band, they will tend to be positively charged near the dislocation core, where the Fermi level will be close to the valence band (bent by the screened charge associated with the dislocation). Further away from the dislocation the point defects are expected to be negatively charged, if they have an acceptor level below the middle of the gap. The total line charge around the dislocation within a radius $\sim D$, which will control the Coulomb interaction between the partials, may now be considerably larger since $R$ in equation (9) for the cloud surrounding the dislocation can be much greater than $b_{T}$. Thus the anomalously large widths of extrinsically dissociated screw dislocations in Ge and Si (5) might be due to coulomb forces; if the extrinsic and intrinsic stacking fault energies are the same, using (8) the large widths can be explained if the partials had a net effective line charge of $\sim^{e} / b_{T}$, of either sign, within a radius $\sim D$. 
At this stage the importance of coulomb forces is uncertain, but in view of the interaction between dislocation and point defects, and the charged states of the latter, the possibility of Coulomb forces and their effect on partial dislocation separation must be borne in mind. The coulomb force effect seems to offer a more plausible explanation for the anomalously wide screw dislocations in $\mathrm{Ge}$ and $\mathrm{Si}$ than the alternative assumption of $\gamma_{E} \sim \frac{1}{2} \gamma_{j}$, since the stacking sequence for both types of fault are interrupted only at the fourth nearest planes /23/. 6. Dissociated dislocations in GaAs. - Gai and Howie /6/ and Gottschalk, Patzer and Alexander /8/ have demonstrated the existence of dissociated dislocations in III- $V$ compounds, the former in GaP, the latter in a number of these compounds. In both cases no account was taken of possible differences between the two types of dislocations ( $\alpha$ and $\beta$ ) with edge components which can be distinguished in these polar structures (Haasen /24/). These have Burgers vectors of opposite sign and differ in the species of atoms

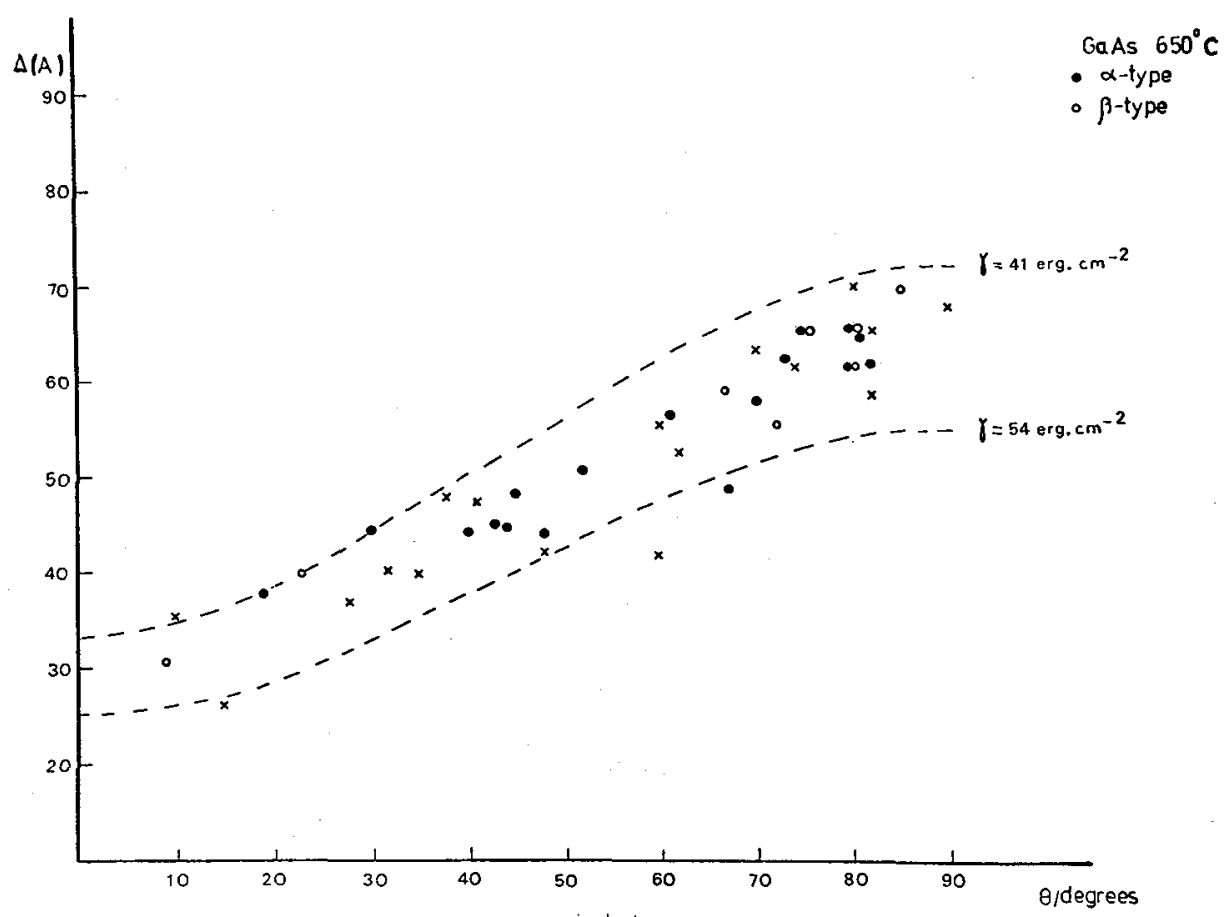

Fig. 6 : Separation of partial dislocations (after correcting image positions) in GaAs as a function of dislocation orientation $\theta$, together with theoretical curves using anisotropic elasticity theory, for values of $Y=41$ and $54 \mathrm{erg} \mathrm{cm}^{-2}$. Full and open circles represent values for $\alpha$ and $\beta$ type dislocations respectively (9); crosses for dislocations the sign of which was not determined. on which the extra half planes terminate, and in their mobilities (Erofeeva and Dsipyan /25/). Weak beam investigations have been carried out on $n$ type GaAs $\left(\mathrm{N}_{\mathrm{d}}-\mathrm{N}_{\mathrm{a}} \sim 2 \times 10^{16} \mathrm{~cm}^{-3}\right)$ with a view to extending the results of (8) to dislocations with a wider range of orientations and to look for possible effects from the polarity on edge dislocations of opposite sign ( $\alpha$ and $\beta$ type dislocations). Figure 6 summarises function of orientation (Gomez and Hirsch /9/). Only dislocations with intrinsic faults were found. The intrinsic stacking fault energy was determined to be $\gamma=48 \pm 6$ erg $\mathrm{cm}^{-2}$, in reasonable agreement with the value obtained in (8) $\left(55 \pm 5 \mathrm{erg} \mathrm{cm}^{-2}\right)$ from dislocations with predominantly edge character. Data for $\alpha$ and $\beta$ dislocations are included (indicated by full and open circles in figure 6 ). Both types of dislocations have the same widths to within the experimental error. These observations suggest that the different core structures of the two types of dislocations which may be responsible for their different mobilities have no effect on the partial dislocation separations.

$\alpha$-type

(1)

(1) the results of partial dislocation separation as a

aAs $650^{\circ} \mathrm{C}$ 


\section{References}

/1/ Cockayne, D.J.H., Ray, I.L.R. and whelan, M.J., Phi1. Mag. 20 (1969) 1265.

/2/ Ray, I.L.R. and Cockayne, D.J.H., Proc. R. Soc. A325. (1971) 534.

/3/ Ray, I.L.R. and Cockayne, D.J.H., J. Microsc. 98 (1973) 170.

14/ Häussermann, F. and Schaumburg, H., Phil. Mag. 27 (1973) 745 .

/5/ Gómez, A., Cockayne, D.J.H., Hirsch, P.B. and Vitek, V., Phil. Mag. 31 (1975) 105.

16/ Gai, P.L. and Howie, A., Phil. Mag. 30 (1974) 939.

17/ Alexander, H., Proc. 6th Europ. Congr. Electron Microscopy, Jerusalern, 1976, p. 208.

18/ Gottschalk, H., Patzer, G. and Alexander, H., Phys. Status Solidi (a) 45 (1978) 207.

19/ Gómez; A. and Hirsch, P.B., Phil. Mag. 38 (1978) 733.

110/ Meingast, R. and Alexander, H., Phys. Status Solidi (a) 17 (1973) 229.

111/ Gomez, A. and Hirsch, P.B., Phil. Mag. 36 (1977) 169.

12/ Hornstra, J., J. Phys. Chem. Solids $\underline{5}$ (1958) 129.

113/ Haasen, P. and Seeger, A., Halbleiterprobleme IV (Braunschweig), 1958, p.68.

/14/ Shockley, W., Phys. Rev. 91 (1953) 228.

115/ Labusch, R. and Schettler, R., Phys. Status SoTidi (a) 9 (1972) 455.

/16/ Labusch, R. and Schröter, W., Lattice Defects in Semiconductors, Inst. Phys. Conf. Ser. No.23 (1975) p.56.

/17/ Wagner, R. and Haasen, P., Lattice Defects in Semiconductors, Inst. Phys. Conf. Ser. No.23 (1975), p.387.

18/ Celli, V., Gold, A. and Thomson, R., Phys. Rev. Lett. 8 (1962) 96 .

19/ Mott, N.F. and Davis, E.A., Electronic Processes in Non-crystalline Solids (Oxford : Clarendon Press) 1971 , p. 123.

/20/ Jones, R., Phit. Mag. 35 (1977) 57.

121/ Springer, E., Diploma Thesis, Göttingen, 1967.

122/ Read, W.T., Phil. Mag. 45 (1954) 775, 1119.

123/ Hirth, J.P. and Lothe, J., Theory of dislocations (New York : McGraw-Hi11) 1968, p.355.

124/ Haasen, P., Acta Metallogr. 5 (1957) 598.

125/ Erofeeva, S.A. and Osipyan, Yu.A., Sov. Phys. Solid State 15 (1973) 538. 